\title{
BDDC AND FETI-DP ALGORITHMS WITH A CHANGE OF BASIS FORMULATION ON ADAPTIVE PRIMAL CONSTRAINTS*
}

\author{
HYEA HYUN KIM ${ }^{\dagger}$, ERIC CHUNG ${ }^{\ddagger}$, AND JUNXIAN WANG ${ }^{\S}$
}

\begin{abstract}
BDDC (Balancing Domain Decomposition by Constraints) and FETI-DP (Dual-Primal Finite Element Tearing and Interconnecting) algorithms with adaptively enriched primal constraints are considered. The coarse component of the two algorithms is built on the set of primal unknowns consisting of those at subdomain vertices and those from the adaptive primal constraints after a change of basis. For the FETI-DP algorithm, a more general form of a preconditioner is proposed to extend the algorithm to the set of primal unknowns including those from the adaptive primal constraints. In addition, it can be shown that the two algorithms share the same spectra except those equal to one or zero when the same set of adaptive primal constraints are employed. Numerical results are included for both two and three dimensional model problems.
\end{abstract}

Key words. FETI-DP, BDDC, adaptive primal constraints, change of basis, condition numbers

AMS subject classifications. 65F10, 65N30, 65N55

1. Introduction. A finite element discretization of the following model elliptic problem is considered,

$$
\int_{\Omega} \rho(x) \nabla u(x) \cdot \nabla v(x) d x=\int_{\Omega} f(x) v(x) d x, \quad \forall v(x) \in H_{0}^{1}(\Omega),
$$

where $\Omega$ is a domain in $\mathbb{R}^{2}$ or $\mathbb{R}^{3}$ and $H_{0}^{1}(\Omega)$ is the space of square integrable functions up to the first weak derivatives with trace equal to zero. The coefficient $\rho(x)$ can be highly heterogeneous across the finite element boundaries. The discrete problem of the above model problem can be efficiently solved iteratively by utilizing domain decomposition preconditioners. In this work, BDDC (Balancing Domain Decomposition by Constraints) and FETI-DP (Dual-Primal Finite Element Tearing and Interconnecting) algorithms with adaptively enriched primal constraints are analyzed under a change of basis formulation. The adaptive primal constraints are introduced to enhance the robustness of the BDDC and FETI-DP preconditioners to the heterogeneous coefficients in the model elliptic problems. We refer to $[4,19,7,2,18]$ for a general introduction to standard BDDC and FETI-DP algorithms and their connections. For the variants of the methods enriched with adaptive primal constraints we refer to $[20,21,22,5$, $23,15,16,14,3,11,13]$. We note that for all such algorithms certain generalized eigenvalue problems are solved to select the set of adaptive primal constraints under a given tolerance value. Estimates for the condition number by the given tolerance value were provided in [14] for the FETI-DP algorithm and in [3,23] for the BDDC algorithm, both in three dimensions. In [13] both algorithms are considered and generalized eigenvalue problems are proposed on face and edge nodal equivalence classes in three dimensions. We refer to [8, 9, 24, 6, 25] for adaptive algorithms in a different framework of domain decomposition methods.

* Received August 30, 2017. Accepted February 4, 2018. Published online on April 26, 2018. Recommended by Susanne Brenner. The first author was supported by the National Research Foundation of Korea(NRF) grants funded by NRF-2015R1A5A1009350, the second author was supported by the Hong Kong RGC General Research Fund (Project 14317516) and the CUHK Direct Grant for Research 2016-17, and the third author was supported by the National Natural Science Foundation of China (Grant No. 11201398) and Open Foundation of Guangdong Provincial Engineering Technology Research Center for Data Science(2016KF07).

$\dagger$ Department of Applied Mathematics and Institute of Natural Sciences, Kyung Hee University, Korea (hhkimekhu.ac.kr).

$\ddagger$ Department of Mathematics, The Chinese University of Hong Kong, Hong Kong SAR (t schungemath. cuhk. edu.hk).

${ }^{\S}$ School of Mathematics and Computational Science, Xiangtan University, Xiangtan, Hunan 411105, China (wangjunxian@xtu.edu.cn). 
In the authors' previous studies [12,13] and other related works [14], the adaptive primal constraints in the FETI-DP algorithm are enforced by using a projection. For the proposed algorithm it was shown that the condition numbers are controlled by a user-defined tolerance value, which is used to select the adaptive primal constraints from generalized eigenvalue problems on each equivalence classes, i.e., on edges and faces. For the BDDC algorithm in $[12,13]$ the adaptive primal constraints can be transformed to explicit unknowns and added to the set of primal unknowns. The standard form of the BDDC algorithm can then be generalized to such a set of primal unknowns with the estimate of condition numbers controlled by the user defined tolerance value. On the other hand, the FETI-DP algorithm with a change of basis formulation to the adaptive primal constraints has a limitation in the analysis of condition numbers. For the proposed preconditioner, one can not obtain the identity $P_{D}=I-E_{D}$ which is used to show the analysis of condition numbers in the standard FETIDP and BDDC methods; see (4.1) for definitions of $E_{D}$ and $P_{D}$, and Lemma 5.2 for their relation. In [13], it was observed that the FETI-DP algorithm presents numerical instability and needs considerable cost for projection when the number of adaptive constraints becomes significant.

In this work, we propose a more general form of a FETI-DP preconditioner which makes it possible to extend the FETI-DP algorithm to the change of basis formulation for the adaptive primal constraints. The change of basis formulation can give a more stable and efficient FETIDP algorithm. For the proposed preconditioner, we can obtain the identity $E_{D}+P_{D}=I$, after the change of basis, for the FETI-DP algorithms as well, and thus, show that the condition numbers of the adaptive BDDC and FETI-DP algorithms with the change of basis formulation are identical. Different from the standard FETI-DP preconditioners, the blocks of subdomain matrices and scaling matrices corresponding to the adaptive primal unknowns will appear in the proposed preconditioner. We note that part of this work was presented at the 24th International Conference on Domain Decomposition Methods and at the same conference an adaptive FETI-DP algorithm with a change of basis formulation was presented by Axel Klawonn, where different generalized eigenvalue problems are introduced and different tools are used in the analysis of condition numbers.

This paper is organized as follows. In Sections 2 and 3, finite element spaces and adaptive BDDC and FETI-DP algorithms are introduced, where a more general form of the FETI-DP preconditioner is proposed. In Section 4, generalized eigenvalue problems are formed on faces and edges to select the effective adaptive primal constraints which make the resulting algorithms robust to the heterogeneous coefficients. In Section 5, the operators $E_{D}$ and $P_{D}$ are shown to satisfy the property $E_{D}+P_{D}=I$, and thus the two algorithms share the same set of eigenvalues except zeros and ones. In Section 6 numerical results are included for both two and three dimensional model problems to show that the two algorithms give the same nonzero extreme eigenvalues.

2. Model problem and finite element spaces. We recall the model problem in (1.1). The domain $\Omega$ can be in two or three dimensions. We introduce a finite element space $\widehat{X}$ for the given domain $\Omega$. For a presentation of BDDC and FETI-DP algorithms, we introduce a non-overlapping subdomain partition $\left\{\Omega_{i}\right\}$, where we assume that the subdomain boundaries do not cut the triangles in the finite element mesh. We use the notation $X_{i}$ to denote the restriction of $\widehat{X}$ to $\Omega_{i}$. Each subdomain $\Omega_{i}$ is then equipped with the finite element space $X_{i}$.

We further introduce $W_{i}$ as the restriction of $X_{i}$ to the subdomain interface unknowns, $W$, and $X$ as the product of local finite element spaces $W_{i}$ and $X_{i}$, respectively. We note that functions in $W$ or $X$ are decoupled across the subdomain interfaces. We then select some primal unknowns among the decoupled unknowns on the subdomain interfaces and enforce that finite element functions are continuous on them. We denote the corresponding spaces by 
$\widetilde{W}$ and $\widetilde{X}$, respectively. We also introduce $\widehat{W}$ as the subspace of $W$, where the unknowns are fully coupled on the subdomain interface.

The preconditioners in BDDC and FETI-DP algorithms will be developed based on the partially coupled space $\widetilde{W}$ and appropriate scaling matrices. In our adaptive methods, we will select primal unknowns from each nodal equivalence classes of subdomain interfaces. In more detail, edges in $2 D$ and faces in $3 D$ are nodal equivalence classes shared by two subdomains, edges in $3 D$ are nodal equivalence classes shared by more than two subdomains, and vertices are end points of edges in both $2 D$ and $3 D$. We refer to [17] for these definitions.

In our approach, we first include the unknowns at subdomain vertices in the set of primal unknowns. Adaptive primal constraints will then be selected from eigenvectors of certain generalized eigenvalue problems on faces and edges using a given tolerance value. The corresponding adaptive primal unknowns are then obtained by applying a change of basis on the adaptively selected primal constraints. These explicit unknowns can then be assembled strongly just as primal unknowns at subdomain vertices in the standard BDDC and FETIDP algorithms. We note that in our previous study a change of basis formulation is only considered and analyzed for the BDDC algorithm. In this work, we will extend the change of basis formulation to the FETI-DP algorithm by introducing a more general form of the FETI-DP preconditioner.

3. Adaptive BDDC and FETI-DP algorithms. We introduce matrices $K_{i}$ and $S_{i}$ for each subdomain $\Omega_{i}$. The matrices $K_{i}$ are obtained from a Galerkin approximation of

$$
a_{i}(u, v)=\int_{\Omega_{i}} \rho(x) \nabla u \cdot \nabla v d x
$$

on finite element spaces $X_{i} . S_{i}$ are the Schur complements of $K_{i}$, obtained from $K_{i}$ by eliminating unknowns interior to $\Omega_{i}$. Let $\widetilde{R}_{i}: \widetilde{W} \rightarrow W_{i}$ be the restriction operator corresponding to $\partial \Omega_{i}$, and let $\widetilde{S}$ be a partially coupled matrix defined by

$$
\widetilde{S}=\sum_{i=1}^{N} \widetilde{R}_{i}^{T} S_{i} \widetilde{R}_{i} .
$$

Let $\widetilde{R}$ be the extension from $\widehat{W}$ to $\widetilde{W}$. The discrete problem of (1.1) from the finite element space $\widehat{X}$ can be reduced to the following interface problem,

$$
\widetilde{R}^{T} \widetilde{S} \widetilde{R} u_{\Gamma}=\widetilde{R}^{T} \widetilde{g}
$$

where $u_{\Gamma}$, in $\widehat{W}$, denotes the restriction of $u$ to the subdomain interface, and $\widetilde{g}$, in $\widetilde{W}$, is the vector obtained from the right-hand side $f(x)$.

In the BDDC algorithm the above matrix equation is solved iteratively by using the following preconditioner,

$$
M_{B D D C}^{-1}=\widetilde{R}^{T} \widetilde{D} \widetilde{S}^{-1} \widetilde{D}^{T} \widetilde{R}
$$

where $\widetilde{D}$ is a scaling matrix of the form

$$
\widetilde{D}=\sum_{i=1}^{N} \widetilde{R}_{i}^{T} D_{i} \widetilde{R}_{i} .
$$

Here the matrices $D_{i}$ are defined for unknowns in $W_{i}$ and they are introduced to resolve heterogeneity in $\rho(x)$ across the subdomain interface. In more details, $D_{i}$ consists of blocks 
$D_{F}^{(i)}, D_{E}^{(i)}, D_{V}^{(i)}$, where $F$ denotes corresponding blocks to unknowns in faces, $E$ to unknowns in edges, and $V$ to unknowns at vertices, respectively. In addition, those blocks satisfy the partition of unity for a given $F, E$, and $V$, respectively.

The FETI-DP algorithm [7, 18, 2] is a dual form of the BDDC algorithm. After the change of basis for the unknowns corresponding to the adaptively selected constraints, we can obtain the resulting FETI-DP algebraic system

$$
B \widetilde{S}^{-1} B^{T} \lambda=d,
$$

where $\widetilde{S}$ is the partially coupled matrix at subdomain vertices and adaptive primal unknowns, and $B$ is the matrix with entries $0,-1$, and 1 , which is used to enforce continuity for the remaining decoupled interface unknowns. We introduce the notation $M$ for the set of Lagrange multipliers $\lambda$. The dimension of $M$ is identical to the number of continuity constraints enforced on the remaining decoupled interface unknowns. The algebraic system (3.1) is then solved by an iterative method with the following preconditioner

$$
M_{F E T I}^{-1}=\sum_{i=1}^{N} B_{D, \Delta}^{(i)} S_{i}\left(B_{D, \Delta}^{(i)}\right)^{T}
$$

where $\left(B_{D, \Delta}^{(i)}\right)^{T}: M \rightarrow W_{i}$ is defined by

$$
\begin{gathered}
\left.\left(B_{D, \Delta}^{(i)}\right)^{T} \lambda\right|_{F}=\operatorname{sign}\left(i, \lambda_{i j}\right) D_{F, \Delta}^{(j)} \lambda_{i j} \text { on each } F \in F(i), \\
\left.\left(B_{D, \Delta}^{(i)}\right)^{T} \lambda\right|_{E}=\sum_{l \in n(E, i)} \operatorname{sign}\left(i, \lambda_{i l}\right) D_{E, \Delta}^{(l)} \lambda_{i l} \text { on each } E \in E(i),
\end{gathered}
$$

and

$$
\left.\left(B_{D, \Delta}^{(i)}\right)^{T} \lambda\right|_{V}=0 \text { on each } V \in V(i) .
$$

Here $F(i), E(i)$, and $V(i)$ denote the set of faces, edges, and vertices of subdomain $\Omega_{i}$, respectively, $n(E, i)$ denotes the set of neighboring subdomain indices sharing the edge $E$ with $\Omega_{i}$, and $\lambda_{i j}$ denotes the part of Lagrange multipliers $\lambda$ used to enforce continuity on the decoupled unknowns across $\Omega_{i}$ and $\Omega_{j}$. In addition, $\operatorname{sign}\left(i, \lambda_{i l}\right)$ are 1 or -1 depending on the sign of $B_{\Delta}^{(i)}$ in $B$ for the corresponding location to $\lambda_{i l}$. The matrices $D_{F, \Delta}^{(j)}$ and $D_{E, \Delta}^{(l)}$ are obtained from blocks of $D_{F}^{(j)}$ and $D_{E}^{(l)}$ as follows:

$$
D_{F, \Delta}^{(j)}=\left[\begin{array}{l}
D_{F, \Delta \Delta}^{(j)} \\
D_{F, \Pi \Delta}^{(j)}
\end{array}\right], \quad D_{E, \Delta}^{(l)}=\left[\begin{array}{l}
D_{E, \Delta \Delta}^{(l)} \\
D_{E, \Pi \Delta}^{(l)}
\end{array}\right],
$$

where the subscripts $\Delta$ and $\Pi$ correspond to blocks in $D_{F}^{(j)}$ and $D_{E}^{(l)}$ related to the decoupled unknowns and the adaptive primal unknowns, respectively, i.e.,

$$
D_{F}^{(j)}=\left[\begin{array}{ll}
D_{F, \Delta \Delta}^{(j)} & D_{F, \Delta \Pi}^{(j)} \\
D_{F, \Pi \Delta}^{(j)} & D_{F, \Pi \Pi}^{(j)}
\end{array}\right], D_{E}^{(l)}=\left[\begin{array}{ll}
D_{E, \Delta \Delta}^{(l)} & D_{E, \Delta \Pi}^{(l)} \\
D_{E, \Pi \Delta}^{(l)} & D_{E, \Pi \Pi}^{(l)}
\end{array}\right] .
$$

Different from the standard FETI-DP preconditioner, the proposed preconditioner contains blocks of the scaling matrices and local Schur complement matrices involving the adaptive 
primal unknowns. With this new form of the FETI-DP preconditioner, we can show that the adaptive FETI-DP algorithm with the change of basis formulation has the same spectra as the corresponding BDDC algorithm except those equal to zero or one. We can thus obtain the same condition number bound as that of the BDDC algorithm. We note that when no adaptive primal unknowns are chosen, this preconditioner is identical to that considered in the standard FETI-DP algorithm.

4. Adaptively enriched primal unknowns. The adaptive constraints will be selected by considering a generalized eigenvalue problem on each nodal equivalence class. The idea is originated from the upper bound estimate of BDDC and FETI-DP preconditioners. We note that the lower bound can be obtained from the partition of unity property of the scaling matrices and it can be shown that the minimum eigenvalue is one $[2,18,26]$. In the estimate of condition numbers of BDDC and FETI-DP algorithms, the average and jump operators are defined as

$$
E_{D}=\widetilde{R} \widetilde{R}^{T} \widetilde{D}, \quad P_{D}=B_{D}^{T} B
$$

where $B=\left(\begin{array}{ll}B_{\Delta} & 0\end{array}\right)$ and $B_{D}^{T}=\left(\begin{array}{lll}B_{D, \Delta}^{(1)} & \cdots & B_{D, \Delta}^{(N)}\end{array}\right)^{T}$. We note that $B: \widetilde{W} \rightarrow M$ and $B_{D}^{T}: M \rightarrow W$; see the definition of $\left(B_{D, \Delta}^{(i)}\right)^{T}$ in (3.2)-(3.4).

The adaptive constraints are treated just as unknowns at subdomain vertices after change of basis formulation in both BDDC and FETI-DP algorithms, i.e., the continuities of them are enforced explicitly. We note that in our previous work one can not get $E_{D}+P_{D}=I$ when the standard FETI-DP preconditioner is considered for the change of basis formulation, i.e., without the blocks from the adaptive primal unknowns in $B_{D}^{T}$.

In the following, we review the generalized eigenvalue problems and the estimate of condition numbers for the adaptive BDDC algorithm proposed in [13]. We first form generalized eigenvalue problems for faces $F$, which are nodal equivalence classes shared by two subdomains. For that we introduce $S_{F}^{(i)}$ to represent the block in $S_{i}$ corresponding to the unknowns interior to $F . \widetilde{S}_{F}^{(i)}$ represents the Schur complement of $S_{i}$ obtained by eliminating unknowns except those interior to $F . \widetilde{S}_{F}^{(i)}$ satisfies the following minimal energy property,

$$
v_{F}^{T} \widetilde{S}_{F}^{(i)} v_{F} \leq v^{T} S_{i} v \text {, for any }\left.v\right|_{F}=v_{F},
$$

where $\left.v\right|_{F}$ denotes the restriction of $v$ to the unknowns interior to $F$. The notation $A: B$ is a parallel sum for symmetric and semi-positive definite matrices $A$ and $B$ defined as, see [1],

$$
A: B=A(A+B)^{+} B,
$$

where $(A+B)^{+}$denotes a pseudo inverse. The parallel sum satisfies the following properties

$$
A: B=B: A, \quad A: B \leq A, \quad A: B \leq B,
$$

and it was first used in [5] when forming generalized eigenvalues problems.

In $3 D$, for a face $F$, the following generalized eigenvalue problem is considered

$$
A_{F} v_{F}=\lambda \widetilde{A}_{F} v_{F},
$$

where

$$
A_{F}=\left(D_{F}^{(j)}\right)^{T} S_{F}^{(i)} D_{F}^{(j)}+\left(D_{F}^{(i)}\right)^{T} S_{F}^{(j)} D_{F}^{(i)}, \widetilde{A}_{F}=\widetilde{S}_{F}^{(i)}: \widetilde{S}_{F}^{(j)}
$$


The eigenvalues are all positive. For a given tolerance $\lambda_{T O L}$, we select eigenvectors $v_{F, l}, l \in$ $N(F)$, corresponding to eigenvalues $\lambda_{l}$ larger than $\lambda_{T O L}$. The following constraints will then be enforced on the unknowns in $F$,

$$
\left(A_{F} v_{F, l}\right)^{T}\left(w_{F}^{(i)}-w_{F}^{(j)}\right)=0, l \in N(F) .
$$

After a change of basis, the above constraints can be transformed into explicit unknowns; see [13] for more implementation details of the change of basis. The explicit unknowns are denoted by $w_{F, \Pi}^{(i)}$ and they are then added to the initial set of primal unknowns. The remaining unknowns are called dual unknowns and are denoted by $w_{F, \Delta}^{(i)}$. In $2 D$, for an edge we can form the generalized eigenvalue problem as in the case of a face in $3 D$. We will use the same notation $F$ to denote an edge in $2 D$.

For the $2 D$ case with only edge and vertex nodal equivalence classes, we can obtain that

$$
\begin{aligned}
& \left\langle\widetilde{S}\left(I-E_{D}\right) \widetilde{w},\left(I-E_{D}\right) \widetilde{w}\right\rangle \leq C \sum_{F}\left(\left\langle A_{F} \widetilde{w}_{F, \Delta}^{(i)}, \widetilde{w}_{F, \Delta}^{(i)}\right\rangle+\left\langle A_{F} \widetilde{w}_{F, \Delta}^{(j)}, \widetilde{w}_{F, \Delta}^{(j)}\right\rangle\right) \\
& \leq C \lambda_{T O L} \sum_{F}\left(\left\langle\widetilde{A}_{F} \widetilde{w}_{F, \Delta}^{(i)}, \widetilde{w}_{F, \Delta}^{(i)}\right\rangle+\left\langle\widetilde{A}_{F} \widetilde{w}_{F, \Delta}^{(j)}, \widetilde{w}_{F, \Delta}^{(j)}\right\rangle\right) \\
& \leq C \lambda_{T O L} \sum_{F}\left(\left\langle S_{i} w_{i}, w_{i}\right\rangle+\left\langle S_{j} w_{j}, w_{j}\right\rangle\right) \\
& \leq C \lambda_{T O L}\langle\widetilde{S} \widetilde{w}, \widetilde{w}\rangle
\end{aligned}
$$

where the estimate on the dual unknowns are bounded by $\lambda_{T O L}$ in the second inequality, and (4.3) and the property of $\widetilde{S}_{F}^{(i)}$ in (4.2) are used in the third inequality. Above, we use $\widetilde{w}_{F, \Delta}^{(i)}$ to represent the vector with value $w_{F, \Delta}^{(i)}$ at the location of dual unknowns and value zero at the location of adaptive primal unknowns.

For an edge $E$ in $3 D$, shared by more than two subdomains, we introduce the following generalized eigenvalue problem,

$$
A_{E} v_{E}=\lambda \widetilde{A}_{E} v_{E}
$$

where

$$
A_{E}=\sum_{m \in I(E)} \sum_{l \in I(E) \backslash\{m\}}\left(D_{E}^{(l)}\right)^{T} S_{E}^{(m)} D_{E}^{(l)}, \quad \widetilde{A}_{E}=\prod_{m \in I(E)} \widetilde{S}_{E}^{(m)} .
$$

Here $I(E)$ denotes the set of subdomain indices sharing the edge $E$, and $\prod_{m \in I(E)} \widetilde{S}_{E}^{(m)}$ is the parallel sum of $\widetilde{S}_{E}^{(m)}$. The matrices $S_{E}^{(m)}$ and $\widetilde{S}_{E}^{(m)}$ are defined similarly as $S_{F}^{(i)}$ and $\widetilde{S}_{F}^{(i)}$. For a given $\lambda_{T O L}$, the eigenvectors with their eigenvalues larger than $\lambda_{T O L}$ will be selected and denoted by $v_{E, l}, l \in N(E)$. The following constraints will be then enforced on the unknowns in $E$,

$$
\left(A_{E} v_{E, l}\right)^{T}\left(w_{E}^{(i)}-w_{E}^{(m)}\right)=0, l \in N(E), m \in I(E) \backslash\{i\} .
$$

Similarly to the face case, the above constraints can be transformed into explicit unknowns after a change of basis. We note that when we form matrices for generalized eigenvalue problems in (4.4) and (4.5), the local Schur complement matrices $S_{F}^{(l)}, \widetilde{S}_{F}^{(l)}, S_{E}^{(l)}$, and $\widetilde{S}_{E}^{(l)}$ are explicitly formed in our algorithm. This adds considerable computational cost to our algorithm compared to the standard BDDC or FEFI-DP methods. To reduce the cost one can form an economic 
version of the Schur complement matrices by solving local problems restricted on slabs of a face $F$ or an edge $E$; see [13, Section 5.2] and [16]. In [14], generalized eigenvalue problems are defined on the closed faces shared by two subdomains and the selected adaptive primal constraints are restricted and enforced on the open faces and open edges. In their method, generalized eigenvalue problems are not needed for edges shared by three subdomains, but are still needed for edges shared by more than three subdomains to enhance the set of adaptive primal constraints.

By using the adaptively selected primal unknowns on each face $F$ and edge $E$ as above, in $3 D$ we can obtain the following estimate

$$
\left\langle\widetilde{S}\left(I-E_{D}\right) \widetilde{w},\left(I-E_{D}\right) \widetilde{w}\right\rangle \leq C \lambda_{T O L}\langle\widetilde{S} \widetilde{w}, \widetilde{w}\rangle
$$

where $C$ is a constant depending on the maximum number of edges and faces per subdomain, and the maximum number of subdomains sharing an edge, but independent of the coefficient $\rho(x)$; see [13]. We note that (4.6) is the key estimate in the analysis of the BDDC algorithm.

5. Analysis of condition number bounds. Using the adaptively enriched primal unknowns described in Section 4 and the estimate in (4.6), we can obtain the following estimate of condition numbers for the BDDC algorithm; see [13].

THEOREM 5.1. The BDDC algorithm with a change of basis formulation for the adaptively chosen set of primal unknowns with a given tolerance $\lambda_{T O L}$ has the following bound for condition numbers,

$$
\kappa\left(M_{B D D C}^{-1} \widetilde{R}^{T} \widetilde{S} \widetilde{R}\right) \leq C \lambda_{T O L},
$$

where $C$ is a constant depending only on $N_{F(i)}, N_{E(i)}, N_{I(E)}$, which are the number of faces per subdomain, the number of edges per subdomain, and the number of subdomains sharing an edge E, respectively.

For the FETI-DP algorithm with the same set of primal unknowns, those at subdomain vertices and those from the adaptive primal constraints after the change of basis, we can show that the FETI-DP algorithm shares the same spectra with the associate BDDC algorithm except zero and one. To obtain the result we first show the following properties of the $P_{D}$ operator defined in (4.1).

LEMmA 5.2. For any $\widetilde{w}$ in $\widetilde{W}, P_{D} \widetilde{w}$ in $W$ has the same values at the location of primal unknowns and thus $P_{D} \widetilde{w}$ is in $\widetilde{W}$. In addition, $P_{D} \widetilde{w}$ satisfies that

$$
\widetilde{R}_{i}\left(\widetilde{w}-E_{D} \widetilde{w}\right)=R_{i}\left(P_{D} \widetilde{w}\right), \quad \forall \widetilde{w} \in \widetilde{W},
$$

where $\widetilde{R}_{i}(\widetilde{w})$ and $R_{i}(w)$ are restrictions of $\widetilde{w} \in \widetilde{W}$ and $w \in W$ to $W_{i}$, respectively.

Proof. Recall the definition of $P_{D}=B_{D}^{T} B$ and $\left(B_{D, \Delta}^{(i)}\right)^{T}$ in (3.2)-(3.4). In the following, we simply use the notation $(w)_{i}$ to denote $R_{i}(w)$ or $\widetilde{R}_{i}(w)$ for $w$ in $W$ or $\widetilde{W}$. On a face $F$ of $\partial \Omega_{i}$, we assume that $F$ is shared with its neighboring subdomain $\Omega_{j}$. We then obtain

$$
\left.\left(P_{D} \widetilde{w}\right)_{i}\right|_{F}=\left[\begin{array}{l}
D_{F, \Delta \Delta}^{(j)}\left(w_{F, \Delta}^{(i)}-w_{F, \Delta}^{(j)}\right) \\
D_{F, \Pi \Delta}^{(j)}\left(w_{F, \Delta}^{(i)}-w_{F, \Delta}^{(j)}\right)
\end{array}\right],\left.\quad\left(P_{D} \widetilde{w}\right)_{j}\right|_{F}=\left[\begin{array}{l}
D_{F, \Delta \Delta}^{(i)}\left(w_{F, \Delta}^{(j)}-w_{F, \Delta}^{(i)}\right) \\
D_{F, \Pi \Delta}^{(i)}\left(w_{F, \Delta}^{(j)}-w_{F, \Delta}^{(i)}\right)
\end{array}\right],
$$

where $\left(P_{D} \widetilde{w}\right)_{i}$ denotes the restriction to $W_{i},\left.w_{i}\right|_{F}$ denotes the restriction of $w_{i}$ to unknowns interior to $F$. From the partition of unity property $D_{F}^{(i)}+D_{F}^{(j)}=I$, we have

$$
D_{F, \Pi \Delta}^{(i)}+D_{F, \Pi \Delta}^{(j)}=0
$$


and thus

$$
D_{F, \Pi \Delta}^{(j)}\left(w_{F, \Delta}^{(i)}-w_{F, \Delta}^{(j)}\right)=D_{F, \Pi \Delta}^{(i)}\left(w_{F, \Delta}^{(j)}-w_{F, \Delta}^{(i)}\right),
$$

which means $P_{D} \widetilde{w}$ has the same value at the location of adaptive primal unknowns in the face $F$.

Similarly, for an edge $E$, we obtain

$$
\begin{aligned}
&\left.\left(P_{D} \widetilde{w}\right)_{i}\right|_{E}= {\left[\begin{array}{ll}
\sum_{l \in I(E)} & D_{E, \Delta \Delta}^{(l)}\left(w_{E, \Delta}^{(i)}-w_{E, \Delta}^{(l)}\right) \\
\sum_{l \in I(E)} & D_{E, \Pi \Delta}^{(l)}\left(w_{E, \Delta}^{(i)}-w_{E, \Delta}^{(l)}\right)
\end{array}\right], } \\
&\left.\left(P_{D} \widetilde{w}\right)_{k}\right|_{E}=\left[\begin{array}{ll}
\sum_{l \in I(E)} D_{E, \Delta \Delta}^{(l)}\left(w_{E, \Delta}^{(k)}-w_{E, \Delta}^{(l)}\right) \\
\sum_{l \in I(E)} D_{E, \Pi \Delta}^{(l)}\left(w_{E, \Delta}^{(k)}-w_{E, \Delta}^{(l)}\right)
\end{array}\right],
\end{aligned}
$$

where $I(E)$ denotes the set of subdomain indices sharing the edge $E$ and the indices $i$ and $k$ are in $I(E)$. By subtracting the primal parts in the above two terms and using $\sum_{l \in I(E)} D_{E, \Pi \Delta}^{(l)}=$ 0 , we obtain

$$
\begin{aligned}
& \sum_{l \in I(E)} D_{E, \Pi \Delta}^{(l)}\left(w_{E, \Delta}^{(i)}-w_{E, \Delta}^{(l)}\right)-\sum_{l \in I(E)} D_{E, \Pi \Delta}^{(l)}\left(w_{E, \Delta}^{(k)}-w_{E, \Delta}^{(l)}\right) \\
& =\left(\sum_{l \in I(E)} D_{E, \Pi \Delta}^{(l)}\right)\left(w_{E, \Delta}^{(i)}-w_{E, \Delta}^{(k)}\right) \\
& =0
\end{aligned}
$$

which shows that $P_{D} \widetilde{w}$ has the same value at the location of adaptive primal unknowns in the edge $E$. Above, we have also used the partition of unity property, $\sum_{l \in I(E)} D_{E}^{(l)}=I$ and thus $\sum_{l \in I(E)} D_{E, \Pi \Delta}^{(l)}=0$.

Since $\left.\left(P_{D} \widetilde{w}\right)_{i}\right|_{V}=0$ for the unknowns at subdomain vertices $V$, by combining the results on the adaptive primal unknowns in faces and edges, we conclude that $P_{D} \widetilde{w}$ has the same value at the location of primal unknowns in different subdomains.

We will now show that $\left(\widetilde{w}-E_{D} \widetilde{w}\right)_{i}=\left(P_{D} \widetilde{w}\right)_{i}$. For that, we first consider $\left(\widetilde{w}-E_{D} \widetilde{w}\right)_{i}$ at dual unknowns on each $F$ of subdomain $\Omega_{i}$ and obtain that

$$
\begin{aligned}
\left.\left(\widetilde{w}-E_{D} \widetilde{w}\right)_{i}\right|_{F, \Delta} & =w_{F, \Delta}^{(i)}-\sum_{k=i, j} D_{F, \Delta \Delta}^{(k)} w_{F, \Delta}^{(k)}-\sum_{k=i, j} D_{F, \Delta \Pi}^{(k)} w_{F, \Pi}^{(k)} \\
& =D_{F, \Delta \Delta}^{(j)}\left(w_{F, \Delta}^{(i)}-w_{F, \Delta}^{(j)}\right) \\
& =\left.\left(P_{D} \widetilde{w}\right)_{i}\right|_{F, \Delta}
\end{aligned}
$$

where we have used $w_{F, \Pi}^{(i)}=w_{F, \Pi}^{(j)}, \sum_{k=i, j} D_{F, \Delta \Delta}^{(k)}=I$, and $\sum_{k=i, j} D_{F, \Delta \Pi}^{(k)}=0$.

Similarly for adaptive primal unknowns on each $F$ of subdomain $\Omega_{i}$, we obtain

$$
\begin{aligned}
\left.\left(\widetilde{w}-E_{D} \widetilde{w}\right)_{i}\right|_{F, \Pi} & =w_{F, \Pi}^{(i)}-\sum_{k=i, j} D_{F, \Pi \Delta}^{(k)} w_{F, \Delta}^{(k)}-\sum_{k=i, j} D_{F, \Pi \Pi}^{(k)} w_{F, \Pi}^{(k)} \\
& =D_{F, \Pi \Delta}^{(j)}\left(w_{F, \Delta}^{(i)}-w_{F, \Delta}^{(j)}\right) \\
& =\left.\left(P_{D} \widetilde{w}\right)_{i}\right|_{F, \Pi}
\end{aligned}
$$

where we also have used $w_{F, \Pi}^{(i)}=w_{F, \Pi}^{(j)}, \sum_{k=i, j} D_{F, \Pi \Pi}^{(k)}=I$, and $D_{F, \Pi \Delta}^{(i)}=-D_{F, \Pi \Delta}^{(j)}$. 
For the dual unknowns on each edge $E$,

$$
\begin{aligned}
\left.\left(\widetilde{w}-E_{D} \widetilde{w}\right)_{i}\right|_{E, \Delta} & =w_{E, \Delta}^{(i)}-\sum_{l \in I(E)} D_{E, \Delta \Delta}^{(l)} w_{E, \Delta}^{(l)}-\sum_{l \in I(E)} D_{E, \Delta \Pi}^{(l)} w_{E, \Pi}^{(l)} \\
& =\sum_{l \in I(E)} D_{E, \Delta \Delta}^{(l)}\left(w_{E, \Delta}^{(i)}-w_{E, \Delta}^{(l)}\right) \\
& =\left.\left(P_{D} \widetilde{w}\right)_{i}\right|_{E, \Delta},
\end{aligned}
$$

where we have used that $w_{E, \Pi}^{(l)}$ have the same value, $\sum_{l \in I(E)} D_{E, \Delta \Pi}^{(l)}=0$, and $\sum_{l \in I(E)} D_{E, \Delta \Delta}^{(l)}=I$.

For the adaptive primal unknowns on each edge $E$,

$$
\begin{aligned}
\left.\left(\widetilde{w}-E_{D} \widetilde{w}\right)_{i}\right|_{E, \Pi} & =w_{E, \Pi}^{(i)}-\sum_{l \in I(E)} D_{E, \Pi \Delta}^{(l)} w_{E, \Delta}^{(l)}-\sum_{l \in I(E)} D_{E, \Pi \Pi}^{(l)} w_{E, \Pi}^{(l)} \\
& =\sum_{l \in I(E)} D_{E, \Pi \Delta}^{(l)}\left(w_{E, \Delta}^{(i)}-w_{E, \Delta}^{(l)}\right) \\
& =\left.\left(P_{D} \widetilde{w}\right)_{i}\right|_{E, \Pi},
\end{aligned}
$$

where we have used that $w_{E, \Pi}^{(l)}$ have the same value, $\sum_{l \in I(E)} D_{E, \Pi \Delta}^{(l)}=0$, and $\sum_{l \in I(E)} D_{E, \Pi \Pi}^{(l)}=I$.

By using Lemma 5.2, we can show that the two algorithms share the same set of spectra except one and zero; see [18, 2]. Combining with the result for the BDDC algorithm in Theorem 5.1, we have

THEOREM 5.3. The FETI-DP algorithm with the change of basis formulation has the bound

$$
\kappa\left(M_{F E T I}^{-1} F_{D P}\right) \leq C \lambda_{T O L},
$$

where $C$ is a constant depending only on $N_{F(i)}, N_{E(i)}, N_{I(E)}$, which are the number of faces per subdomain, the number of edges per subdomain, and the number of subdomains sharing an edge $E$, respectively.

6. Numerical results. In our experiments, we consider a unit square or cubic domain $\Omega$ and divide it into a uniform mesh with grid size $h$. We partition the mesh into uniform square or cubic subdomains, or into irregular subdomains by the METIS mesh partitioner [10]. We use $H$ to denote the size of the subdomains in the case of uniform square or cubic partition and $N_{d}$ to denote the number of subdomains in a partition. In the conjugate gradient method for solving the system, the iteration is stopped when the relative residual norm is below $10^{-10}$.

6.1. 2D examples. In this section, we consider two dimensional model problems. For a given subdomain partition and given tolerance value, we first include the unknowns at subdomain vertices to the set of primal unknowns and then enrich the initial set of primal unknowns by including adaptive primal unknowns selected from the generalized eigenvalue problem on each edge under the given tolerance value.

In the first example, we consider a model problem with $\rho(x)$ having both channels and inclusions of high contrast as shown in Figure 6.1. For this model, we present in Table 6.1 the results of the two algorithms when the domain is partitioned into uniform square subdomains with a given local problem size $H / h$. Consistent with our theory, the two algorithms have the same nonzero extreme eigenvalues. The two algorithms are quite robust with respect to the 


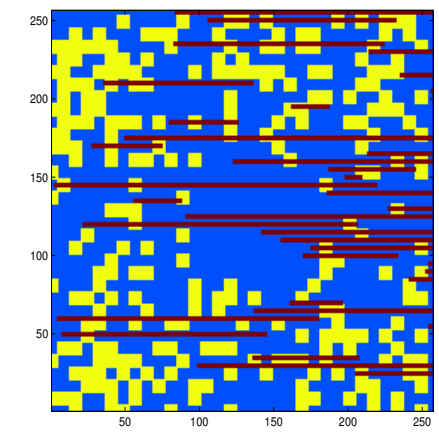

FIG. 6.1. $\rho(x)$ with channels and inclusions: channel $\left(\rho(x)=10^{3}\right)$, inclusion $\left(\rho(x)=10^{-3}\right)$, and elsewhere $(\rho(x)=1)$.

TABLE 6.1

Performance of adaptive BDDC (Bddc) and FETI-DP $(F d p)$ with $\lambda_{T O L}=1+\log (H / h)$ for $\rho(x)$ as in Figure 6.1 with both channels and inclusions: $\lambda_{\min }$ (minimum eigenvalues), $\lambda_{\max }$ (maximum eigenvalues), Iter (number of iterations), pnumF (total number of adaptive primal unknowns), and $p F$ (number of adaptive primal unknowns per edge).

\begin{tabular}{c|c||c|c|c|c|c}
$N_{d}(H / h)$ & method & $\lambda_{\min }$ & $\lambda_{\max }$ & Iter & pnumF & $\mathrm{pF}$ \\
\hline $8^{2}$ & Bddc & 1.00 & 3.11 & 13 & 178 & 1.58 \\
$(H / h=32)$ & Fdp & 1.00 & 3.11 & 13 & 178 & 1.58 \\
\hline $16^{2}$ & Bddc & 1.00 & 1.75 & 11 & 604 & 1.25 \\
$(H / h=16)$ & Fdp & 1.00 & 1.75 & 11 & 604 & 1.25 \\
\hline $32^{2}$ & Bddc & 1.00 & 1.47 & 8 & 2098 & 1.05 \\
$(H / h=8)$ & Fdp & 1.00 & 1.47 & 9 & 2098 & 1.05
\end{tabular}

coefficient $\rho(x)$, even with only less than two adaptive primal unknowns per edge. We can also observe that the minimum eigenvalues are identical to one and the maximum eigenvalues follow the tolerance value $\lambda_{T O L}$.

In the second example, we consider a more challenging case with random and high contrast value $\rho(x)$ in the range $\left(10^{-3}, 10^{3}\right)$. The value $\rho(x)$ is piecewise constant at each finite element. In Tables 6.2 and 6.3, the performance of the two algorithms is presented by increasing local problem size $H / h$ for a given uniform subdomain partition and by increasing the number of subdomains in the uniform partition for a fixed local problem size $H / h$. As in the previous example, we observe that the minimum eigenvalues are all one and the two algorithms have the same maximum eigenvalues. In addition, for this highly heterogeneous case we can control the condition numbers by using only less than two adaptive primal unknowns per edge.

In Table 6.4, the results of the two algorithms are presented for the same $\rho(x)$ considered in the previous two tables, when irregular subdomain partitions as in Figure 6.2 are used. We again observe that the two algorithms have the same extreme eigenvalues and they perform well even for the quite irregular subdomain partitions, which shows the practicality of the proposed scheme in real applications.

6.2. 3D examples. We consider three dimensional model problems in this section. Unknowns at subdomain vertices are included in the set of primal unknowns first, and generalized eigenvalue problems defined in (4.4) and (4.5) are then used to introduce additional 


\section{ETNA}

Kent State University and

Johann Radon Institute (RICAM)

TABLE 6.2

Performance of adaptive BDDC and FETI-DP with $\lambda_{T O L}=1+\log (H / h)$ for random $\rho(x)$ in $\left(10^{-3}, 10^{3}\right)$ by increasing $H / h$ in a fixed subdomain partition $N_{d}=3^{2}: \lambda_{\min }$ (minimum eigenvalues), $\lambda_{\max }$ (maximum eigenvalues), Iter (number of iterations), pnumF (total number of adaptive primal unknowns), and pF (number of adaptive primal unknowns per edge).

\begin{tabular}{c|c||c|c|c|c|c}
$H / h$ & method & $\lambda_{\min }$ & $\lambda_{\max }$ & Iter & pnumF & $\mathrm{pF}$ \\
\hline 6 & Bddc & 1.00 & 1.30 & 7 & 17 & 1.41 \\
& Fdp & 1.00 & 1.30 & 7 & 17 & 1.41 \\
\hline 12 & Bddc & 1.00 & 1.68 & 9 & 23 & 1.91 \\
& Fdp & 1.00 & 1.68 & 9 & 23 & 1.91 \\
\hline 18 & Bddc & 1.00 & 1.81 & 9 & 21 & 1.75 \\
& Fdp & 1.00 & 1.81 & 9 & 21 & 1.75 \\
\hline 24 & Bddc & 1.00 & 2.16 & 10 & 23 & 1.91 \\
& Fdp & 1.00 & 2.16 & 10 & 23 & 1.91 \\
\hline 30 & Bddc & 1.00 & 2.63 & 10 & 20 & 1.66 \\
& Fdp & 1.00 & 2.63 & 10 & 20 & 1.66
\end{tabular}

TABLE 6.3

Performance of adaptive BDDC and FETI-DP with $\lambda_{T O L}=1+\log (H / h)$ for random $\rho(x)$ in $\left(10^{-3}, 10^{3}\right)$ by increasing $N_{d}$ and with a fixed $H / h=16: \lambda_{\min }$ (minimum eigenvalues), $\lambda_{\max }$ (maximum eigenvalues), Iter (number of iterations), pnumF (total number of adaptive primal unknowns), and $p F$ (number of adaptive primal unknowns per edge).

\begin{tabular}{c|c||c|c|c|c|c}
$N_{d}$ & method & $\lambda_{\min }$ & $\lambda_{\max }$ & Iter & pnumF & $\mathrm{pF}$ \\
\hline $4^{2}$ & Bddc & 1.00 & 1.74 & 10 & 42 & 1.75 \\
& Fdp & 1.00 & 1.74 & 11 & 42 & 1.75 \\
\hline $8^{2}$ & Bddc & 1.00 & 3.11 & 16 & 189 & 1.68 \\
& Fdp & 1.00 & 3.11 & 16 & 189 & 1.68 \\
\hline $16^{2}$ & Bddc & 1.00 & 2.69 & 16 & 805 & 1.67 \\
& Fdp & 1.00 & 2.69 & 17 & 805 & 1.67
\end{tabular}

adaptive primal unknowns on faces and edges using the tolerance values $\lambda_{T O L}^{F}=1+$ $\log (H / h), \lambda_{T O L}^{E}=1000$, respectively. We note that in [13] it was observed that eigenvalues from generalized eigenvalue problems on edges are much larger than those on faces and by choosing larger tolerance values for edges one can choose less and still effective adaptive constraints. Much larger eigenvalues are obtained for edges since the matrix $\widetilde{A}_{E}$ in the generalized eigenvalue problem on an edge, see (4.5), is involved by more subdomains than $\widetilde{A}_{F}$ on a face, see (4.4). This makes the right-hand side matrix less optimal for the edge case. In our experiment, we thus choose different tolerance values for face and edge cases.

We first consider the model with coefficient distribution $\rho(x)$ shown in Figure 6.3. The performance of adaptive BDDC and FETI-DP methods is presented in Table 6.5 by increasing $H / h$ in a fixed subdomain partition $N_{d}=3^{3}$. We can see that both methods have the same set of primal unknowns, maximum eigenvalues, nearly the same minimum eigenvalues (which are equal to one) and iteration counts except a small numerical perturbation. The number of adaptive primal unknowns per face and per edge are relatively robust with respect to $H / h$ and in our test case about two per face and one per edge are chosen. The condition numbers and iteration counts are also robust given the high contrast in the coefficient $\rho(x)$.

We next consider highly varying and random coefficients $\rho(x)=10^{r}$, where $r$ is chosen randomly from $(-3,3)$ for each finite element. The results are listed in Table 6.6 with an increasing $N_{d}=N^{3}$ and a fixed $H / h=12$. We observe the same performance as in the previous case that both methods have the same set of primal unknowns, maximum eigenvalues, 


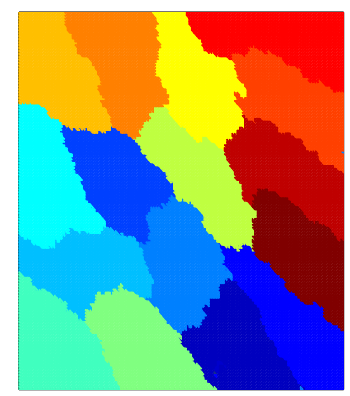

FIG. 6.2. An example of irregular subdomain partition with $N_{d}=16$ and $1 / h=256$.

TABLE 6.4

Performance of adaptive BDDC and FETI-DP for random $\rho(x)$ in $\left(10^{-3}, 10^{3}\right)$ and on irregular subdomain partitions: $N_{d}$ (number of subdomains), $h$ (element size), $\lambda_{\text {min }}$ (minimum eigenvalues), $\lambda_{\text {max }}$ (maximum eigenvalues), Iter (number of iterations), pnumF (total number of adaptive primal unknowns), $p F$ (number of adaptive primal unknowns per edge), $n_{F}$ (maximum number of nodes per edge), and $\lambda_{T O L}=1+\log \left(n_{F}\right)$.

\begin{tabular}{c|c||c|c|c|c|c|c|c}
$N_{d}$ & $1 / h$ & method & $\lambda_{\min }$ & $\lambda_{\max }$ & Iter & pnumF & $\mathrm{pF}$ & $n_{F}$ \\
\hline 16 & 64 & Bddc & 1.00 & 2.69 & 13 & 44 & 1.37 & 39 \\
& & Fdp & 1.00 & 2.69 & 13 & 44 & 1.37 & 39 \\
\hline 64 & \multirow{2}{*}{129} & Bddc & 1.00 & 3.71 & 17 & 235 & 1.45 & 39 \\
& & Fdp & 1.00 & 3.71 & 18 & 235 & 1.45 & 39 \\
\hline 256 & 256 & Bddc & 1.00 & 3.14 & 17 & 1059 & 1.53 & 22 \\
& & Fdp & 1.00 & 3.14 & 18 & 1059 & 1.53 & 22 \\
\hline \hline 16 & \multirow{2}{*}{256} & Bddc & 1.00 & 3.43 & 15 & 37 & 1.12 & 83 \\
& & Fdp & 1.00 & 3.43 & 15 & 37 & 1.12 & 83 \\
\hline 64 & \multirow{2}{*}{256} & Bddc & 1.00 & 3.17 & 17 & 213 & 1.33 & 45 \\
& & Fdp & 1.00 & 3.17 & 17 & 213 & 1.33 & 45 \\
\hline 256 & 256 & Bddc & 1.00 & 3.14 & 17 & 1059 & 1.53 & 22 \\
& & Fdp & 1.00 & 3.14 & 18 & 1059 & 1.53 & 22
\end{tabular}

TABLE 6.5

Performance of adaptive BDDC (Bddc) and FETI-DP (Fdp) for the problem with channels and inclusions $\rho(x)$ as in Figure 6.3 by increasing $H / h$ in a fixed subdomain partition $N_{d}=3^{3}$ and with $\lambda_{\text {TOL }}^{F}=1+$ $\log (H / h), \lambda_{\text {TOL }}^{E}=1000: \lambda_{\min }$ (minimum eigenvalues), $\lambda_{\max }$ (maximum eigenvalues), Iter (number of iterations), pnumF (total number of adaptive primal unknowns on faces), and pnumE (total number of adaptive primal unknowns on edges). $p F$ and $p E$ are the number of adaptive primal unknowns per face and per edge, respectively.

\begin{tabular}{c|c||c|c|c|c|c|c|c}
$H / h$ & method & $\lambda_{\min }$ & $\lambda_{\max }$ & Iter & pnumF & pnumE & $p F$ & $p E$ \\
\hline 4 & Bddc & 1.00 & 1.32 & 9 & 61 & 36 & 1.13 & 1.00 \\
& Fdp & 1.00 & 1.32 & 9 & 61 & 36 & 1.13 & 1.00 \\
\hline 8 & Bddc & 1.00 & 1.61 & 11 & 84 & 36 & 1.56 & 1.00 \\
& Fdp & 1.01 & 1.61 & 11 & 84 & 36 & 1.56 & 1.00 \\
\hline \multirow{2}{*}{12} & Bddc & 1.00 & 1.94 & 12 & 87 & 38 & 1.61 & 1.06 \\
& Fdp & 1.01 & 1.94 & 13 & 87 & 38 & 1.61 & 1.06 \\
\hline 16 & Bddc & 1.00 & 2.24 & 14 & 88 & 40 & 1.63 & 1.11 \\
& Fdp & 1.01 & 2.24 & 14 & 88 & 40 & 1.63 & 1.11
\end{tabular}




\section{ETNA}

Kent State University and

Johann Radon Institute (RICAM)

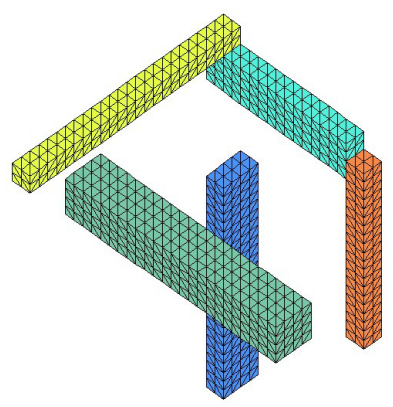

(a) Channels with $\rho(x)=10^{3}$

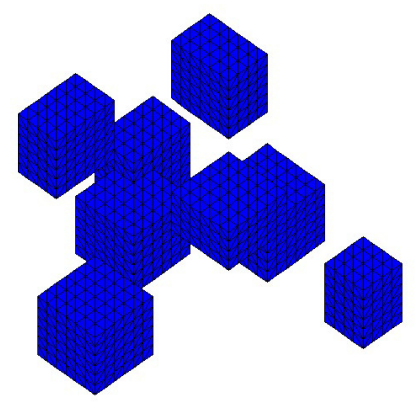

(b) Inclusions with $\rho(x)=10^{-3}$

FIG. 6.3. Coefficient distribution with channels and inclusions: channels $\left(\rho(x)=10^{3}\right)$, small cubes $(\rho(x)=$ $\left.10^{-3}\right)$, and elsewhere $(\rho(x)=1)$.

TABLE 6.6

Performance of adaptive BDDC and FETI-DP with $\lambda_{T O L}^{F}=1+\log (H / h)$ and $\lambda_{T O L}^{E}=1000$ for highly varying and random $\rho(x)$ in $\left(10^{-3}, 10^{3}\right)$ by increasing $N_{d}$ and with a fixed $H / h=12: \lambda_{\min }$ (minimum eigenvalues), $\lambda_{\max }$ (maximum eigenvalues), Iter (number of iterations), pnumF (total number of adaptive primal unknowns on faces), and pnumE (total number of adaptive primal unknowns on edges). $p F$ and $p E$ are the number of adaptive primal unknowns per face and per edge, respectively.

\begin{tabular}{c|c||c|c|c|c|c|c|c}
$N_{d}$ & method & $\lambda_{\min }$ & $\lambda_{\max }$ & Iter & pnumF & pnumE & $p F$ & $p E$ \\
\hline $2^{3}$ & Bddc & 1.01 & 3.15 & 15 & 46 & 18 & 3.83 & 3.00 \\
& Fdp & 1.00 & 3.15 & 16 & 46 & 18 & 3.83 & 3.00 \\
\hline $3^{3}$ & Bddc & 1.01 & 5.11 & 20 & 190 & 115 & 3.52 & 3.19 \\
& Fdp & 1.00 & 5.11 & 21 & 190 & 115 & 3.52 & 3.19 \\
\hline $4^{3}$ & Bddc & 1.01 & 5.83 & 23 & 533 & 320 & 3.70 & 2.96 \\
& Fdp & 1.00 & 5.83 & 24 & 533 & 320 & 3.70 & 2.96
\end{tabular}

nearly the same minimum eigenvalues (which are equal to one) and iteration counts. About four per face and three per edge are chosen as adaptive primal unknowns. The condition numbers and iteration counts increase very mildly as $N_{d}$ increases. The performance of the two methods is presented in Table 6.7 with an increasing $H / h$ in a fixed subdomain partition $N_{d}=3^{3}$, which is similar to the performance in Table 6.5 . We observe that the percentages of primal unknowns on face and edge both decrease despite the fact that the number of primal unknowns per face and edge increases. For example, for $H / h=12$, the percentages of primal unknowns on face and edge are $3.52 / 121=2.91 \%$ and $3.19 / 11=29 \%$, respectively, and for $H / h=16$, those are $1.95 \%$ and $27 \%$.

Finally, for unstructured subdomain partitions as in Figure 6.4, we present the results for $\rho(x)=1$ in Table 6.8, and in Table 6.9 the results for highly varying and random coefficients $\rho(x)=10^{r}$, where $r$ is chosen randomly from $(-3,3)$ at each tetrahedron finite element. We also observe a similar performance as in the previous cases. We choose only one adaptive primal unknown per face and per edge for $\rho(x)=1$ and a little more than one for random $\rho(x)$ under quite irregular subdomain partitions. It means that the two algorithms also perform well for both irregular subdomain partitions and highly heterogeneous coefficients in three dimensions.

7. Conclusion. In this paper, we consider BDDC and FETI-DP algorithms with adaptively enriched primal constraints and analyze their performance under a change of basis formulation. The adaptive primal constraints are introduced to enhance the robustness of 


\section{ETNA}

Kent State University and

Johann Radon Institute (RICAM)

TABLE 6.7

Performance of adaptive BDDC and FETI-DP for the problem with random $\rho(x)$ in $\left(10^{-3}, 10^{3}\right)$ by increasing $H / h$ in a fixed subdomain partition $N_{d}=3^{3}$ and with $\lambda_{\text {TOL }}^{F}=1+\log (H / h), \lambda_{\text {TOL }}^{E}=1000: \lambda_{\min }($ minimum eigenvalues), $\lambda_{\max }$ (maximum eigenvalues), Iter (number of iterations), pnumF (total number of adaptive primal unknowns on faces), and pnumE (total number of adaptive primal unknowns on edges). $p F$ and $p E$ are the number of adaptive primal unknowns per face and per edge, respectively.

\begin{tabular}{c|c||c|c|c|c|c|c|c}
$H / h$ & method & $\lambda_{\min }$ & $\lambda_{\max }$ & Iter & pnumF & pnumE & $p F$ & $p E$ \\
\hline 4 & Bddc & 1.00 & 2.34 & 11 & 91 & 56 & 1.69 & 1.56 \\
& Fdp & 1.00 & 2.34 & 11 & 91 & 56 & 1.69 & 1.56 \\
\hline 8 & Bddc & 1.00 & 2.53 & 15 & 147 & 83 & 2.72 & 2.31 \\
& Fdp & 1.00 & 2.53 & 16 & 147 & 83 & 2.72 & 2.31 \\
\hline 12 & Bddc & 1.01 & 5.11 & 20 & 190 & 115 & 3.52 & 3.19 \\
& Fdp & 1.00 & 5.11 & 21 & 190 & 115 & 3.52 & 3.19 \\
\hline 16 & Bddc & 1.01 & 5.42 & 22 & 237 & 146 & 4.39 & 4.06 \\
& Fdp & 1.00 & 5.42 & 23 & 237 & 146 & 4.39 & 4.06
\end{tabular}

the BDDC and FETI-DP preconditioners for solving elliptic problems with heterogeneous coefficients. The change of basis formulation can enhance the stability and efficiency of the algorithms. In particular, we show that the identity $E_{D}+P_{D}=I$ holds for both algorithms using the proposed change of basis. Hence, we show that the condition numbers of the adaptive BDDC and FETI-DP algorithms with the change of basis formulation are identical. Some numerical examples in both 2D and 3D with both structured and unstructured subdomain partitions are presented to demonstrate the robustness of the algorithms.

\section{REFERENCES}

[1] W. N. Anderson, JR. And R. J. Duffin, Series and parallel addition of matrices, J. Math. Anal. Appl., 26 (1969), pp. 576-594.

[2] S. C. BRENNER AND L.-Y. SUNG, BDDC and FETI-DP without matrices or vectors, Comput. Methods Appl. Mech. Engrg., 196 (2007), pp. 1429-1435.

[3] J. G. CALVO AND O. B. WIDLUND, An adaptive choice of primal constraints for BDDC domain decomposition algorithms, Electron. Trans. Numer. Anal., 45 (2016), pp. 524-544.

http://etna.ricam.oeaw.ac.at/vol.45.2016/pp524-544.dir/pp524-544.pdf

[4] C. R. DoHRMANN, A preconditioner for substructuring based on constrained energy minimization, SIAM J. Sci. Comput., 25 (2003), pp. 246-258.

[5] C. Dohrmann AND C. PeChSTEIn, Modern domain decomposition solvers - BDDC, deluxe scaling, and an algebraic approach, Talk by C. Pechstein at the University Linz, December 2013. http://people.ricam.oeaw.ac.at/c.pechstein/pechstein-bddc2013.pdf

[6] V. Dolean, F. Nataf, R. Scheichl, AND N. SpIllane, Analysis of a two-level Schwarz method with coarse spaces based on local Dirichlet-to-Neumann maps, Comput. Methods Appl. Math., 12 (2012), pp. 391-414.

[7] C. Farhat, M. Lesoinne, P. LeTallec, K. Pierson, And D. RiXen, FETI-DP: a dual-primal unified FETI method. I. A faster alternative to the two-level FETI method, Internat. J. Numer. Methods Engrg., 50 (2001), pp. 1523-1544.

[8] J. GALVIS AND Y. EFENDIEV, Domain decomposition preconditioners for multiscale flows in high-contrast media, Multiscale Model. Simul., 8 (2010), pp. 1461-1483.

[9] - Domain decomposition preconditioners for multiscale flows in high contrast media: reduced dimension coarse spaces, Multiscale Model. Simul., 8 (2010), pp. 1621-1644.

[10] G. Karypis, R. Aggarwal, K. Schoegel, V. Kumar, and S. Shekhar, G. Karypis, R. Aggarwal, AND K. SCHOEGEL, METIS home page.

http://glaros.dtc.umn.edu/gkhome/views/metis

[11] H. H. KIM AND E. CHUNG, A BDDC algorithm with enriched coarse spaces for two-dimensional elliptic problems with oscillaroty and high constrast coefficients, Multiscale Model. Simul., 13 (2015), pp. 571593.

[12] H. H. KIM, E. CHUNG, AND J. WANG, BDDC and FETI-DP methods with enriched coarse spaces for elliptic problems with oscillatory and high contrast coefficients, in Domain Decomposition Methods in Science 


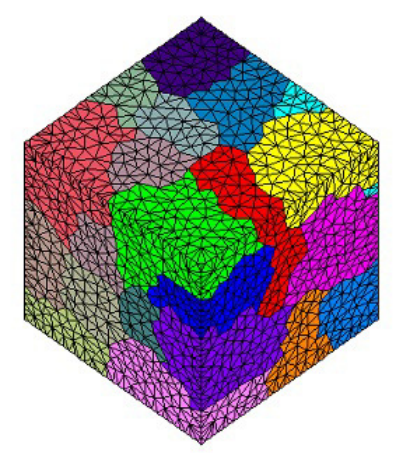

(a) METIS decomposition

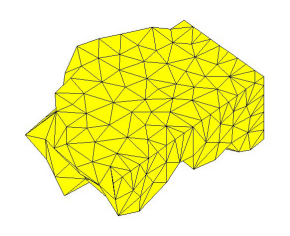

(b) One of the METIS subdomains

FIG. 6.4. Domain decomposition obtained by METIS for the unit cube, $N_{d}=27$ and $h=1 / 20$.

and Engineering XXIII, C.-O. Lee, X.-C. Cai, D. E. Keyes, H. H. Kim, A. Klawonn, E.-J. Park, and O. B. Widlund, eds., vol. 116 of Lect. Notes Comput. Sci. Eng., Springer, Heidelberg, 2017, pp. 179-186.

[13] - BDDC and FETI-DP preconditioners with adaptive coarse spaces for three-dimensional elliptic problems with oscillatory and high contrast coefficients, J. Comput. Phys., 349 (2017), pp. 191-214.

[14] A. KLAWONN, M. KÜHN, AND O. RHEINBACH, Adaptive coarse spaces for FETI-DP in three dimensions, SIAM J. Sci. Comput., 38 (2016), pp. A2880-A2911.

[15] A. KLAWONN, P. RADTKE, AND O. RHEINBACH, FETI-DP with different scalings for adaptive coarse spaces, PAMM Proc. Appl. Math. Mech., 14 (2014), pp. 835-836.

[16] - A comparison of adaptive coarse spaces for iterative substructuring in two dimensions, Electron. Trans. Numer. Anal., 45 (2016), pp. 75-106.

http://etna.ricam.oeaw.ac.at/vol.45.2016/pp75-106.dir/pp75-106.pdf

[17] A. KLAWONn AND O. B. WIDLUnd, Dual-primal FETI methods for linear elasticity, Comm. Pure Appl. Math., 59 (2006), pp. 1523-1572.

[18] J. Li AND O. B. WIDLUND, FETI-DP, BDDC, and block Cholesky methods, Internat. J. Numer. Methods Engrg., 66 (2006), pp. 250-271.

[19] J. MANDEL, C. R. Dohrmann, AND R. TEZAur, An algebraic theory for primal and dual substructuring methods by constraints, Appl. Numer. Math., 54 (2005), pp. 167-193.

[20] J. MANDEL AND B. SOUSEDÍK, Adaptive coarse space selection in the BDDC and the FETI-DP iterative substructuring methods: optimal face degrees of freedom, in Domain Decomposition Methods in Science and Engineering XVI, O. B. Widlund and D. E. Keyes, eds., vol. 55 of Lect. Notes Comput. Sci. Eng., Springer, Berlin, 2007, pp. 421-428.

[21] - Adaptive selection of face coarse degrees of freedom in the BDDC and the FETI-DP iterative substructuring methods, Comput. Methods Appl. Mech. Engrg., 196 (2007), pp. 1389-1399.

[22] J. MAndel, B. Sousedík, AND J. Šístek, Adaptive BDDC in three dimensions, Math. Comput. Simulation, 82 (2012), pp. 1812-1831.

[23] C. Pechstein And C. R. Dohrmann, A unified framework for adaptive BDDC, Electron. Trans. Numer. Anal., 46 (2017), pp. 273-336. http://etna.ricam.oeaw.ac.at/vol.46.2017/pp273-336.dir/pp273-336.pdf

[24] N. Spillane, V. Dolean, P. Hauret, F. Nataf, C. Pechstein, and R. Scheichl, A robust two-level domain decomposition preconditioner for systems of PDEs, C. R. Math. Acad. Sci. Paris, 349 (2011), pp. $1255-1259$. 
ETNA

Kent State University and

Johann Radon Institute (RICAM)

TABLE 6.8

Performance of adaptive BDDC and FETI-DP for $\rho(x)=1$ on unstructured subdomain partitions (see Figure 6.4) and with $\lambda_{\text {TOL }}^{F}=1+\log \left(\min _{i}\left\{H_{i} / h\right\}\right), \lambda_{T O L}^{E}=1000\left(h\right.$ (mesh size), $\left.H_{i}=\max _{x_{1}, x_{2} \in \bar{\Omega}_{i}}\left\{\left|x_{1}-x_{2}\right|\right\}\right)$ : $N_{d}$ (number of subdomains), $\lambda_{\min }$ (minimum eigenvalues), $\lambda_{\max }$ (maximum eigenvalues), Iter (number of iterations), pnumF (total number of adaptive primal unknowns on faces), and pnumE (total number of adaptive primal unknowns on edges). $p F$ and $p E$ are the number of adaptive primal unknowns per face and per edge, respectively.

\begin{tabular}{c|c||c|c|c|c|c|c|c|c}
$N_{d}$ & method & $1 / h$ & $\lambda_{\min }$ & $\lambda_{\max }$ & Iter & pnumF & pnumE & $p F$ & $p E$ \\
\hline 8 & Bddc & 10 & 1.00 & 1.15 & 7 & 12 & 7 & 1.00 & 1.00 \\
& & 20 & 1.00 & 1.37 & 9 & 19 & 17 & 1.00 & 1.00 \\
& & 40 & 1.00 & 1.57 & 10 & 21 & 22 & 1.00 & 1.00 \\
& & 80 & 1.00 & 2.27 & 13 & 22 & 28 & 1.00 & 1.00 \\
& Fdp & 10 & 1.00 & 1.15 & 7 & 12 & 7 & 1.00 & 1.00 \\
& & 20 & 1.00 & 1.37 & 9 & 19 & 17 & 1.00 & 1.00 \\
& & 40 & 1.00 & 1.57 & 11 & 21 & 22 & 1.00 & 1.00 \\
& & 80 & 1.00 & 2.27 & 14 & 22 & 28 & 1.00 & 1.00 \\
\hline 27 & Bddc & 10 & 1.00 & 1.11 & 6 & 10 & 7 & 1.00 & 1.00 \\
& & 20 & 1.00 & 1.26 & 8 & 75 & 53 & 1.00 & 1.00 \\
& & 40 & 1.00 & 1.62 & 10 & 99 & 107 & 1.02 & 1.00 \\
& & 80 & 1.00 & 1.92 & 12 & 108 & 134 & 1.00 & 1.00 \\
& Fdp & 10 & 1.01 & 1.11 & 7 & 10 & 7 & 1.00 & 1.00 \\
& & 20 & 1.00 & 1.27 & 9 & 75 & 53 & 1.00 & 1.00 \\
& & 40 & 1.01 & 1.62 & 11 & 99 & 107 & 1.02 & 1.00 \\
& & 80 & 1.01 & 1.92 & 13 & 108 & 134 & 1.00 & 1.00 \\
\hline 64 & Bddc & 10 & 1.00 & 1.04 & 4 & 2 & 1 & 1.00 & 1.00 \\
& & 20 & 1.00 & 1.29 & 8 & 148 & 73 & 1.00 & 1.00 \\
& & 40 & 1.00 & 1.46 & 9 & 271 & 279 & 1.00 & 1.00 \\
& & 80 & 1.00 & 1.90 & 12 & 302 & 397 & 1.00 & 1.00 \\
& \multirow{3}{*}{ Fdp } & 10 & 1.01 & 1.04 & 4 & 2 & 1 & 1.00 & 1.00 \\
& 20 & 1.01 & 1.29 & 9 & 148 & 73 & 1.00 & 1.00 \\
& & 40 & 1.01 & 1.47 & 10 & 271 & 279 & 1.00 & 1.00 \\
& & 80 & 1.01 & 1.90 & 13 & 302 & 397 & 1.00 & 1.00
\end{tabular}

[25] N. Spillane, V. Dolean, P. Hauret, F. Nataf, And D. J. Rixen, Solving generalized eigenvalue problems on the interfaces to build a robust two-level FETI method, C. R. Math. Acad. Sci. Paris, 351 (2013), pp. 197-201.

[26] A. Toselli And O. Widlund, Domain Decomposition Methods-Algorithms and Theory, Springer, Berlin, 2005 . 
TABLE 6.9

Performance of adaptive BDDC and FETI-DP for random $\rho(x)$ in $\left(10^{-3}, 10^{3}\right)$ on unstructured subdomain partitions (see Figure 6.4) and with $\lambda_{T O L}^{F}=1+\log \left(\min _{i}\left\{H_{i} / h\right\}\right), \lambda_{T O L}^{E}=1000$ (h (mesh size), $H_{i}=$ $\left.\max _{x_{1}, x_{2} \in \bar{\Omega}_{i}}\left\{\left|x_{1}-x_{2}\right|\right\}\right): N_{d}$ (number of subdomains), $\lambda_{\min }$ (minimum eigenvalues), $\lambda_{\max }$ (maximum eigenvalues), Iter (number of iterations), pnumF (total number of adaptive primal unknowns on faces), and pnumE (total number of adaptive primal unknowns on edges). $p F$ and $p E$ are the number of adaptive primal unknowns per face and per edge, respectively.

\begin{tabular}{|c|c|c|c|c|c|c|c|c|c|}
\hline$N_{d}$ & method & $1 / h$ & $\lambda_{\min }$ & $\lambda_{\max }$ & Iter & pnumF & pnumE & $p F$ & $p E$ \\
\hline \multirow[t]{8}{*}{8} & \multirow[t]{4}{*}{ Bddc } & 10 & 1.00 & 1.79 & 10 & 13 & 8 & 1.08 & 1.14 \\
\hline & & 20 & 1.00 & 2.10 & 12 & 24 & 17 & 1.26 & 1.00 \\
\hline & & 40 & 1.00 & 3.20 & 15 & 24 & 31 & 1.14 & 1.41 \\
\hline & & 80 & 1.00 & 4.33 & 19 & 28 & 34 & 1.27 & 1.21 \\
\hline & \multirow[t]{4}{*}{ Fdp } & 10 & 1.00 & 1.79 & 10 & 13 & 8 & 1.08 & 1.14 \\
\hline & & 20 & 1.00 & 2.10 & 13 & 24 & 17 & 1.26 & 1.00 \\
\hline & & 40 & 1.00 & 3.20 & 15 & 24 & 31 & 1.14 & 1.41 \\
\hline & & 80 & 1.00 & 4.33 & 20 & 28 & 34 & 1.27 & 1.21 \\
\hline \multirow[t]{8}{*}{27} & \multirow[t]{4}{*}{ Bddc } & 10 & 1.00 & 1.51 & 8 & 12 & 8 & 1.20 & 1.14 \\
\hline & & 20 & 1.00 & 2.36 & 13 & 87 & 56 & 1.16 & 1.06 \\
\hline & & 40 & 1.01 & 4.91 & 18 & 123 & 126 & 1.27 & 1.18 \\
\hline & & 80 & 1.01 & 4.86 & 19 & 142 & 160 & 1.31 & 1.19 \\
\hline & \multirow[t]{4}{*}{ Fdp } & 10 & 1.02 & 1.51 & 9 & 12 & 8 & 1.20 & 1.14 \\
\hline & & 20 & 1.00 & 2.36 & 13 & 87 & 56 & 1.16 & 1.06 \\
\hline & & 40 & 1.00 & 4.91 & 19 & 123 & 126 & 1.27 & 1.18 \\
\hline & & 80 & 1.00 & 4.86 & 20 & 142 & 160 & 1.31 & 1.19 \\
\hline \multirow[t]{8}{*}{64} & \multirow[t]{4}{*}{ Bddc } & 10 & 1.00 & 1.08 & 4 & 2 & 1 & 1.00 & 1.00 \\
\hline & & 20 & 1.00 & 1.99 & 12 & 158 & 78 & 1.07 & 1.07 \\
\hline & & 40 & 1.01 & 2.81 & 16 & 307 & 309 & 1.13 & 1.11 \\
\hline & & 80 & 1.01 & 5.38 & 19 & 359 & 471 & 1.19 & 1.19 \\
\hline & \multirow[t]{4}{*}{ Fdp } & 10 & 1.00 & 1.08 & 4 & 2 & 1 & 1.00 & 1.00 \\
\hline & & 20 & 1.00 & 1.99 & 12 & 158 & 78 & 1.07 & 1.07 \\
\hline & & 40 & 1.00 & 2.81 & 17 & 307 & 309 & 1.13 & 1.11 \\
\hline & & 80 & 1.00 & 5.38 & 21 & 359 & 471 & 1.19 & 1.19 \\
\hline
\end{tabular}

